\title{
Considerations in Medicine
}

\section{lain Mclnnes}

\section{Correspondence to} lain McInnes, Institute of Infection, Immunity and Inflammation, University of Glasgow, Scotland, UK, iain. mcinnes@glasgow.ac.uk
As clinicians, every day we face decisions requiring us to consider our options based upon the best available data. Whether this is selecting the right medicine for a patient's condition or understanding a medicine's safety profile, it is important to balance the range of options and to determine that which is optimal in the circumstances. Considerations in Medicine provides a succinct synthesis of contemporary clinical topics, considers available data to support decisions, and discusses such decisions in the context of current knowledge and possible future directions for research.

Each edition of Considerations in Medicine focuses on a single topic and considers it from different angles, exploring what we know, what we do not know, and highlights important outstanding questions. Each topic is considered by a panel of international experts who review and discuss the latest information and consider its implications for patient care and future research. The papers published within the journal reflect the output of these in-depth discussions. We seek, thereby, to assist clinicians in making decisions in daily clinical practice and to inform priority areas of research.

Considerations in Medicine is supported by online Continuing Medical Education allowing readers to evaluate their understanding of the topics discussed.

\section{To consider (verb) - Oxford English dictionary}

- 'To think carefully about (something), typically before making a decision'

- 'To think about and be drawn towards la course of action).

- 'To take (something) into account when making a judgement'

This edition of Considerations in Medicine considers the important topic of the use of biosimilar medicines, especially in inflammatory diseases. The advent of biologic medicines has transformed disciplines across inflammation medicine. The arrival of biosimilars raises many issues around health economics, clinical effectiveness, the safety and ethical acceptability of switching, the governance of such strategies, and ensuring long term benefits are maintained. All of these issues are considered in detail but via a highly accessible format. I commend the assembled articles to you. 